\title{
The Road to Unlocking During the COVID-19 Pandemic has Many Potholes Ahead
}

\author{
Mike Stedman ${ }^{1}$, Mark Davies $^{2}$, Mark Lunt $^{2}$, Arpana Verma ${ }^{3}$, and Adrian Heald ${ }^{4}$ \\ ${ }^{1}$ Res Consortium \\ ${ }^{2}$ Affiliation not available \\ ${ }^{3}$ The University of Manchester \\ ${ }^{4}$ Salford Royal Hospitals NHS Trust
}

June 4, 2020

\begin{abstract}
The Facts COVID-19 is the disease associated with the 2019 novel coronavirus Severe Acute Respiratory Syndrome Coronavirus2 (SARS-CoV-2). The pandemic related to this virus has transformed life for billions of people across the globe. Using population based data from England, we recently showed a strong independent relation over time between our calculated $\mathrm{R}$ value for COVID-19 transmission and the number of cases identified as definitely positive in the population. We determined that $26.8 \%$ of the population of the United Kingdom (UK) possibly had been infected by late April 2020 approximating to 18.2 million people. Reflection Different modelling approaches give different answers to the question of how many people in the UK and elsewhere have actually been infected by COVID-19, which all would agree in epidemiological terms is behaving very differently from other Coronaviruses that we have seen in the past. We are learning more and more about this virus. National policy is changing rapidly and COIVD-19 antibody testing is increasingly being applied. This will facilitate better datasets from population based studies. The picture will become clearer as more data accrues over time.
\end{abstract}

\section{The Road to Unlocking During the COVID-19 Pandemic has Many Potholes Ahead}

Mike Stedman ${ }^{1}$, Mark Davies ${ }^{1}$, Mark Lunt ${ }^{2}$, Arpana Verma ${ }^{3}$, Adrian H Heald ${ }^{4,5}$

${ }^{1}$ Res Consortium, Andover, Hampshire; ${ }^{2}$ University of Manchester, Division of Musculoskeletal and Dermatological Sciences; ${ }^{3}$ Population Health, Health Services Research and Primary Care, The University of Manchester, Manchester, United Kingdom; ${ }^{4}$ Department of Diabetes and Endocrinology, Salford; ${ }^{5}$ The Faculty of Biology, Medicine and Health and Manchester Academic Health Sciences Centre, University of Manchester, UK

Key Words: COVID-19, Unlock, Epidemiology, Strategy

Word Count: 896

Number of figures: 0

Corresponding Author: Dr Adrian Heald, Department of Diabetes and Endocrinology, Salford Royal Hospital, Salford, UK

Telephone: +441612065157

Email: adrian.heald@manchester.ac.uk

What is already known about this topic 
COVID-19 is the disease associated with the 2019 novel coronavirus Severe Acute Respiratory Syndrome Coronavirus-2 (SARS-CoV-2). The pandemic related to this virus has transformed life for billions of people across the globe.

Using population-based data from England, we recently showed an independent association over time between our calculated R-value for COVID-19 transmission and the number of cases identified as definitely positive in the population. We estimated based on that methodology that around $26.8 \%$ of the population of the UK may have been infected by late April 2020 approximating to 18.2 million people.

\section{What this article adds}

Different modelling approaches have considered the question of how many people in the UK and elsewhere may have actually been infected by COVID-19.

We are learning more and more about this virus. National policy is changing rapidly and will be enhanced with COVID-19 antibody testing is increasingly being applied. This will facilitate better datasets from population-based studies. The picture will become clearer as more data accrues over time.

\section{Letter to the Editor}

\section{Why we wrote our recently published paper in IJCP}

COVID-19 is the disease associated with the 2019 novel coronavirus Severe Acute Respiratory Syndrome Coronavirus-2 (SARS-CoV-2). The pandemic related to this virus has transformed life for billions of people across the world, with significant perturbation of the global economy.

The work that underpinned our recently published article estimating the proportion of people in the United Kingdom (UK) potentially exposed and infected with COVID-19 (1) was started in mid-April 2020, 4 weeks after the initiation of the lockdown in the UK and the United States of America (USA). Our aim was to find insights and connections from publicly available data at the level of the UK Local Authority, with drivers for the average daily infection rate $\mathrm{R}_{\mathrm{ADIR}}(2)$. The literature shows that many different models are in use today with differing assumptions and various degrees of uncertainty around the estimates derived.

\section{Where the data came from}

Publicly available data covering local population characteristics was collected from a number of sources for the 149 different England Upper Tier Local Authority Areas (UTLAs) (3). Local infection rate is a product of the local population characteristics, social distancing constraints and historic case immunity. All of England is being managed under similar social distancing rules.

\section{What we found}

In establishing an $\mathrm{R}_{\mathrm{ADIR}}$ value based on cases we were helped, during the period that we evaluated, by the UK National Health Service (NHS) in England operating on a testing strategy to confirm a diagnosis in symptomatic individuals. With a third of tests in symptomatic individuals being positive over the period studied, we made the assumption that positive tests reflected a small consistent more severe proportion of the total infected cohort.

We showed an independent relation over time between our calculated $R_{\text {ADIR }}$ value and the number of cases identified as definitely positive in the population. We then inferred by extrapolation using a linear regression model that by late April, $26.8 \%$ of the total population were possibly infected (1). We did not seek to establish an absolute proportion of the total infected number for the population.

\section{The 'Cambridge' Model}

An eminent team of statisticians at the MRC Biostatistics Unit (BSU), University of Cambridge, are collaborating with Public Health England (PHE) regularly to 'nowcast' and forecast COVID-19 infections and deaths (4). This information feeds directly to the Special Advisory Group for Emergencies (SAGE) sub-group, Scientific Pandemic Influenza sub-group on Modelling (SPI-M), and to regional PHE teams, 
The group have estimated the total infected population at 6,540,000 (12\%). The findings also indicate an transmission rate $\mathrm{R}=0.4$ in London likely due to many more cases of infection at around $20 \%$ of the population - in other words the London results appear to lead the national trend. In contrast, the South-West of England with $\mathrm{R}=0.76$ only $5 \%$ of that population are reported as having been infected.

As the Cambridge MRC BSU based their model on deaths which occur around 3 weeks after the MRC Biostatistics Unit (the report is dated $10^{\text {th }}$ May 2020), the originating infections were occurring around the middle of April 2020. This time period coincides with the period to which our own published model applies.

The low R-value in now being seen in London likely relates to the conurbation being at the forefront of the pandemic with a large number of confirmed cases even prior to UK Lockdown on 23 March 2020. Nevertheless, it is reasonable that there are a large number of unreported cases in London, sufficient to reduce the R-value to the level now being seen.

\section{Alternative approaches}

An alternative approach for estimating the spread of the COVID-19 virus undertaken by Dimdore-Miles and Miles (5) used predictive mathematical modeling for the UK and other countries and applied this to daily new cases of the virus. As did our projection, they favoured high values for the number of people infected but asymptomatic, with a multiplier of approximately 200 on the diagnosed cases of COVID-19 in late April 2020, to derive a total population estimate of infection (5). The authors, acknowledged that the result is very sensitive to whether the transmission rate of the virus is different for symptomatic and asymptomatic cases, something about which there is significant uncertainty. The differences between the estimates given by different models, illustrate how difficult it is to estimate the spread of the COVID-19 virus until very large samples of the population can be tested, as is now happening in the UK.

Nevertheless the fact that the Office of National Statistics (ONS) weekly results (6) suggest that around $80 \%$ of people testing positive for COVID-19 have no symptoms suggests that COVID-19 infections in many cases have a minimal impact on health, as severe / life threatening as the infection may be in others.

\section{Conclusion}

Different modelling approaches give different answers to the question of how many people in the UK and elsewhere have actually been infected by COVID-19, which all would agree in epidemiological terms is behaving very differently from other Coronaviruses that we have seen in the past. We have the ability through working collaboratively to build a scientific evidence base for tackling this and future pandemics.

We are learning more and more about this virus. National policy is changing rapidly, and COVID-19 antibody testing is increasingly being applied (7). This will facilitate better datasets from population-based studies. The picture will become clearer as more data accrues over time.

\section{Disclaimers}

Neither Patients nor the public was involved in the design, or conduct, or reporting, or dissemination of our findings.

All authors contributed equally in relation to the writing of this paper. No author has any conflict of interest.

The manuscript is an honest, accurate, and transparent account of the study being reported. No important aspects of the study have been omitted.

\section{References}

1. Stedman M, Davies M, Lunt M, Verma A, Anderson SG, Heald AH. A phased approach to unlocking during the COVID-19 pandemic - Lessons from trend analysis. Int J Clin Pract. 2020;00:e13528. https://doi. org/10.1111/ijcp.13528: Accessed 16 May 2020

2. Van den Driessche P. Reproduction numbers of infectious disease models. Infect Dis Model. 2017; 2: 288-303 
3. LSOAs link to individual UTLA. https://geopo rtal.stati stics.gov.uk/ datas ets/lower -layer -super -outpu t-area-2011-to-upper -tier-local -autho ritie s-2019-looku p-in-engla nd-and-wales -/data: Accessed 15 May, 2020.

4. Birrell P, Blake J, van Leeuwen E, De Angelis D, Joint PHE Modelling Cell, and MRC Biostatistics Unit COVID-19 Working Group. 10 May 2020 https://www.mrc-bsu.cam.ac.uk/no: Accessed 15 May 2020

5. Dimdore-Miles O and David Miles D. Assessing the Spread of the Novel Coronavirus In The Absence of Mass Testing. Covid Economics Edition 16. Published 11 May 2020 athttps://cepr.org/sites/default/files/news/CovidEconomics16.pdf: accessed 15 May 2020

6. https://www.ons.gov.uk/peoplepopulationandcommunity/healthandsocialcare/conditionsanddiseases/bulletins/corona Accessed 31 May 2020

7. Loeffelholz MJ, Tang YW. Laboratory diagnosis of emerging human coronavirus infections - the state of the art. Emerg Microbes Infect 2020; 9: 747-756 